\title{
Concentrating Red Beet Juice Using Osmotic Distillation: Effects of Device Structure
}

\author{
Mahdi Mahdavi ${ }^{1}$, Hossein Mirsaeedghazi ${ }^{2 *}$, Akbar Arabhosseini $^{1}$, Fatemeh Azarikia ${ }^{2}$ \\ 1- Department of Agrotechnology, College of Abouraihan, University of Tehran, Pakdasht, Iran \\ 2- Department of Food Technology, College of Abouraihan, University of Tehran, Pakdasht, Iran
}

\section{A B S T R A C T}

Background and Objectives: Red beet juice, a potential source of valuable nutrients, is widely used as a colorant in food industries. In recent years, to avoid reduction of nutritional values of the red beet during concentration by thermal processing, an osmotic distillation (OD) technique has been suggested. Therefore, the major aim of the present study was to investigate effects of the flow rate, volume of feed and brine solutions and membrane active area on efficiency of the membrane concentration process of red beet juice.

Materials and Methods: In this study, osmotic distillation process was used in a laboratory scale unit equipped with a hydrophobic polyvinylidene fluoride (PVDF) membrane with a pore size of $0.22 \mu \mathrm{m}$ at $25{ }^{\circ} \mathrm{C}$ after centrifuging red beet juice at 4,000 rpm for $17 \mathrm{~min}$. Furthermore, to investigate effects of feed and brine solution volumes on efficacy of the concentration process, two channel heights $(2 \mathrm{~cm}$ and $2 \mathrm{~mm}$ in both feed and brine sections) were tested.

Results: Based on the results, the high feed volume was more effective than the high brine volume for the increasing soluble solid content (SSC) of juice in a constant time. Moreover, it was found that if the process time included more than three hours, the membrane with a higher active area $\left(131.75 \mathrm{~cm}^{2}\right)$ significantly improved total soluble solid content of juice, compared to the membrane with a lower active area $\left(87.83\right.$ and $\left.43.91 \mathrm{~cm}^{2}\right)$. Results showed that a higher volume and flow rate of both feed and brine included had the most desirable effects on concentration efficiency. Based on the findings, flux of the total soluble solid content rise can be considered as a practical parameter to study efficiency of the osmotic distillation.

Conclusion: Efficiency of the concentration process of red beet juice using osmotic distillation can increase by the modification of module configuration and process setup.

Keywords: Membrane concentration, Osmotic distillation, Red beet juice

\section{Introduction}

Red beet (Beta vulgaris L.) juice, an antioxidant for the prevention of cancers and cardiovascular diseases $(1-3)$, is widely used in food industries as a natural colorant source. The major components of the red beet extract include polyphenols and betalains, which are responsible for its antioxidant activity $(1,4)$. In the last decades, there has been a growing interest for the concentration of extracts because this can reduce costs of packaging and storage. However, the heat sensitivity of nutraceutical ingredients in red beet extracts (betalains and polyphenols) makes these extracts inappropriate for concentrating by the thermal process $(3,5)$. Therefore, non-thermal treatments seem to be appropriate options for the prevention of nutraceuticals degeneration during the concentration process.
Osmotic distillation (OD) is a membrane technique usually used at room temperature and normal pressure to concentrate heat-sensitive fluids; hence, it is expected to make negligible chemical changes (6-9). In OD technique, a hydrophobic membrane separates two phases with various concentrations, which include various vapor pressures $(10,11)$. These two phases include juice as the feed and brine as the stripping solution, which move in each side of the membrane. The stripping solution is diluted when water vapor passes through the membrane and hence results in juice concentration $(12,13)$. Several studies have been carried out focusing on membrane concentration of fruit juices using OD. Examples include orange, blood orange, apple, pomegranate and kiwi juices $(11,14$, 
15). Based on the results of these studies, membrane concentration can preserve quality and nutritional values such as antioxidant activity, color and aroma of the juices in contrast to thermal process. Although the geometric structure of membrane module is an important parameter in efficiency of the concentration process, to the best of the authors' knowledge, no studies have been carried out on efficiency of the membrane geometric structure on performance of the concentration process during OD of the fruit juices. Therefore, the aim of the current study was to investigate effects of membrane active area and channel height of the feed and stripping solution on efficiency of the osmotic distillation during membrane concentration of the red beet juice. This study also completes a previous study by the authors, in which, effects of the membrane and thermal concentration processes on the nutritional values of red beet concentrates were compared (16).

\section{Materials and Methods}

Preparation of the red beet extract: Red beet of the cultivar Detroit Dark Red was purchased from a local market in Tehran, Iran. After washing, peeling and slicing, the extract was prepared using juicer (Pars Khazar, Iran). For clarification, the extract was centrifuged at 4,000 rpm for $17 \mathrm{~min}$. Calcium chloride was purchased from Merck (Darmstadt, Germany).

Osmotic distillation (OD) unit: The OD process was carried out in a laboratory scale unit at $25{ }^{\circ} \mathrm{C}$. The unit was equipped with a $0.22-\mu \mathrm{m}$ hydrophobic polyvinylidene fluoride (PVDF) membrane (Millipore, USA) (Fig. 1). Calcium chloride solution $\left(\mathrm{CaCl}_{2}, 4.3\right.$ M, PubChem CID: 5284359) was used as brine. The solution was concentrated using evaporator. The feed tank was fixed on a digital balance (EK-1500, A\&D, Japan) to measure water vapor flux during the membrane process. Moreover, brine and juice were recycled via separate peristaltic pump. To investigate effects of feed and brine solution volumes on efficiency of the concentration process, two channel heights $(2 \mathrm{~cm}$ and $2 \mathrm{~mm}$ in both feed and brine sections) were used. The membrane module was vertically designed to guarantee that feed and brine channels were filled.

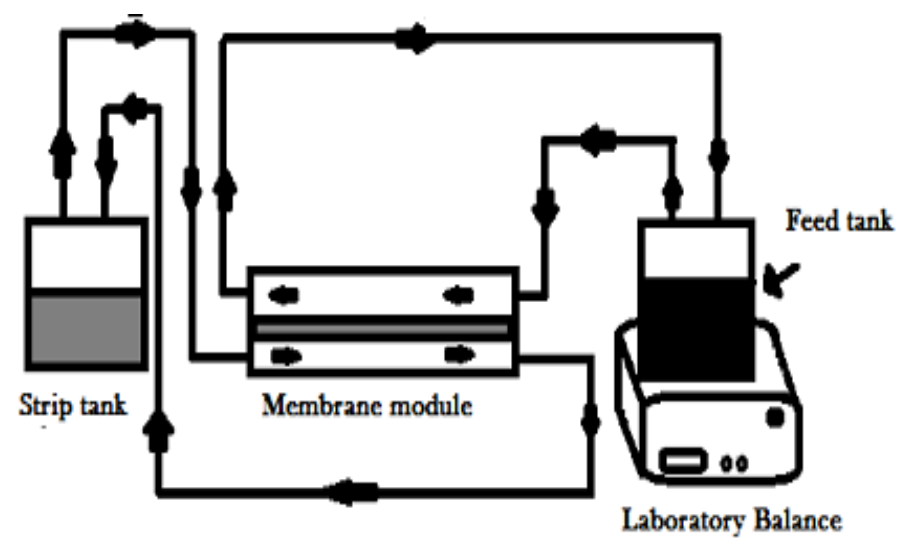

Fig. 1. Scheme of the osmotic distillation unit

Statistical analysis: Statistical analysis of the data was carried out using one-way ANOVA. Duncan's multiple range tests were used to calculate significant differences between the mean values using Minitab Software v.15, (Minitab, State College, PA). All tests were carried out at least twice and the mean values were reported.

\section{Results}

Effects of experiment setup on efficiency of the membrane concentration: Based on the results, higher volumes of feed and brine resulted further reduction in feed weight, compared to that lower volumes of feed and brine did (Fig. 2). It was found that further feed weight loss was linked to further concentration of the red beet juice (Fig. 3). Consequently, membrane concentration efficiency of the red beet juice increased with increased feed and brine volumes. As stated previously, OD was carried out in two module configurations to assess the most effective condition for processing. Results showed that increased feed channel height positively affected the feed weight reduction during membrane concentration of the red beet juice. However, increased brine channel height included negative effects (Fig. 4). Figure 5 illustrates associations between the high feed and brine volumes and the change of soluble solid contents (SSC) of the red beet extract as a function of time. As seen in the figure, comparison of high feed volume with high brine volume showed that high feed volume included considerably further effects on enhancement of the juice SSC at a constant time. Moreover, the concentration process was carried out at various feed and brine flow rates to investigate their effects on efficiency of the red beet concentration process. 


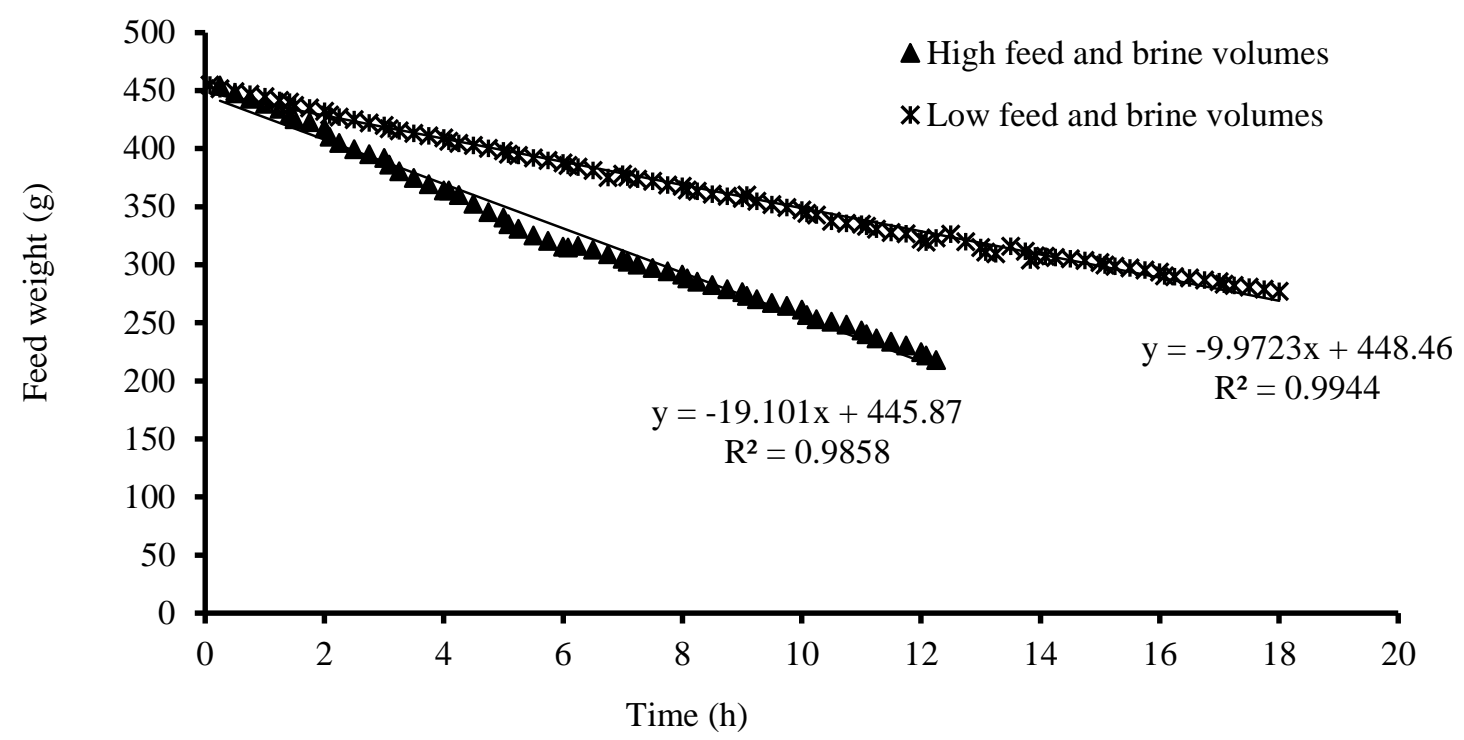

Fig. 2. Effects of feed and brine volumes on the feed weight during membrane concentration of the red beet juice

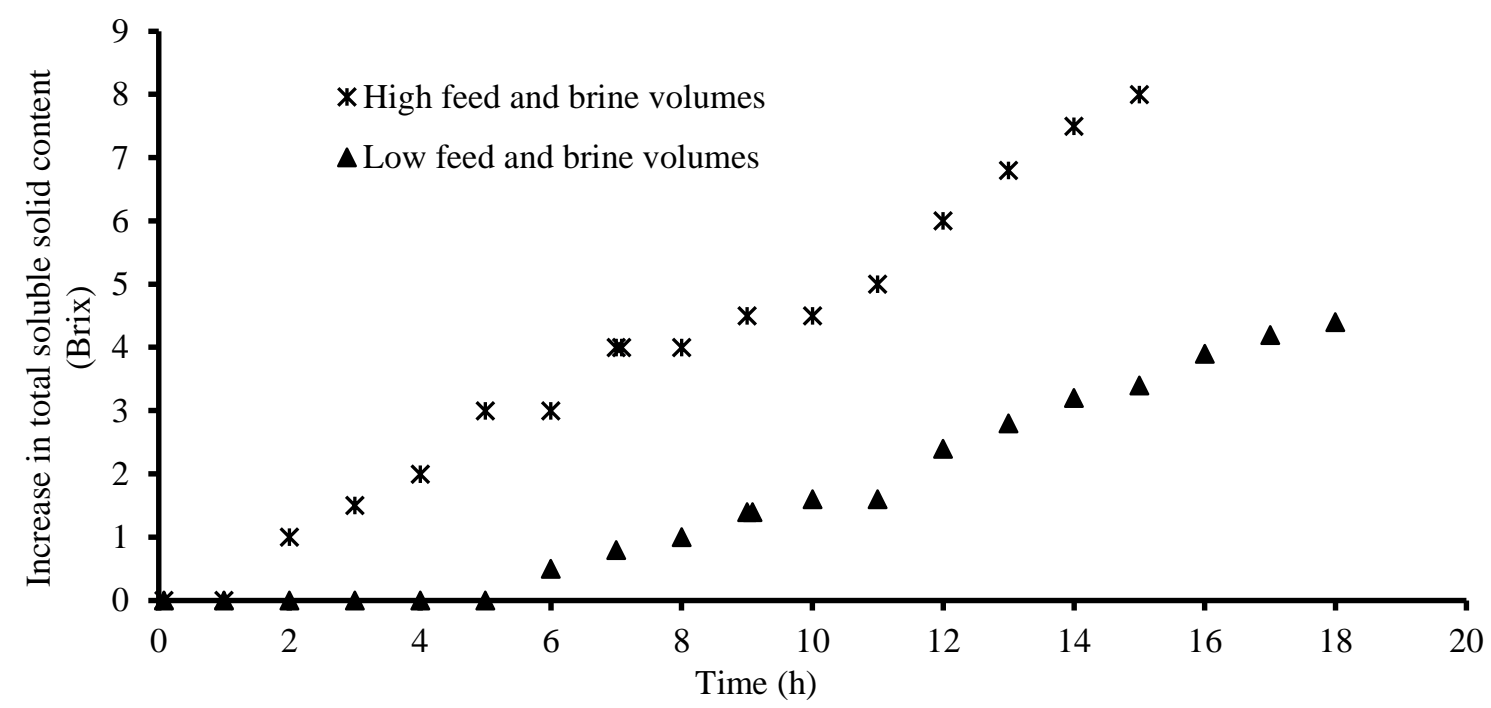

Fig. 3. Effects of feed and brine volumes on feed soluble solid content during membrane concentration of the red beet juice

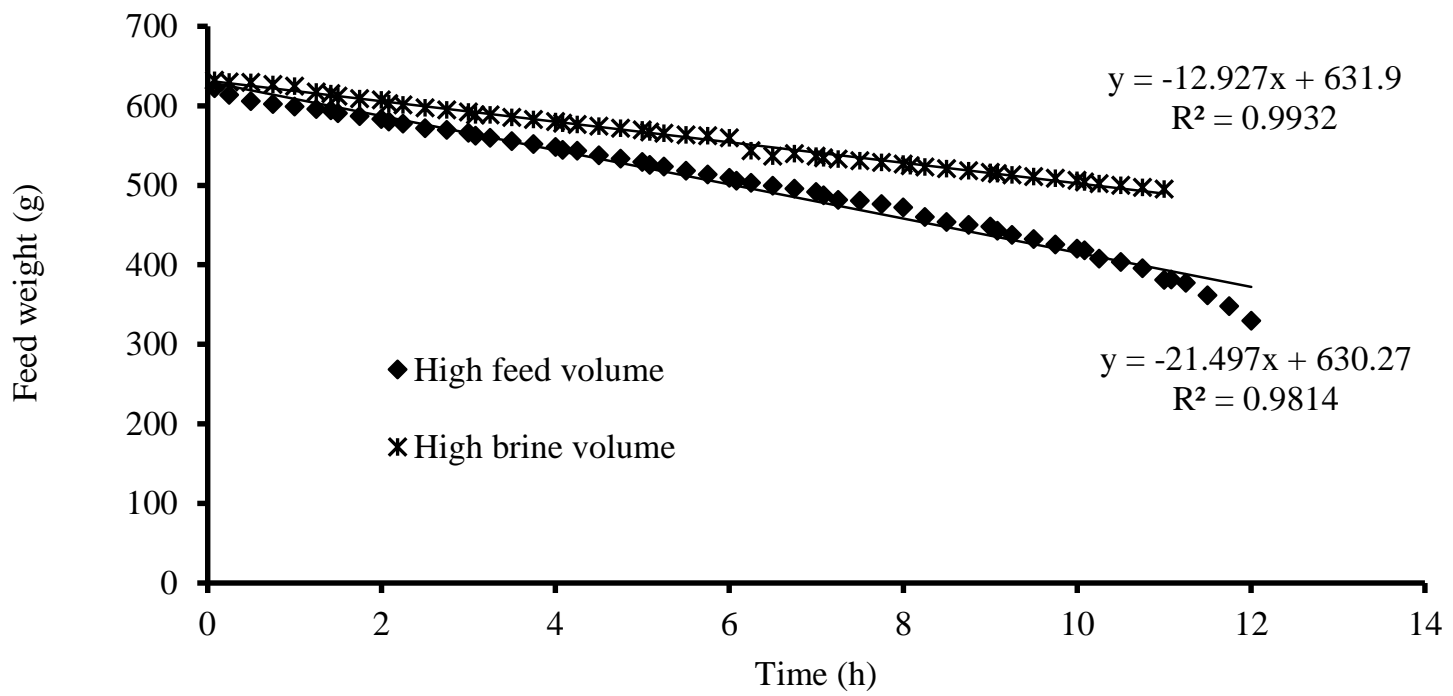

Fig. 4. Effects of feed and brine volumes on the reduction of feed weight during membrane concentration of the red beet juice 


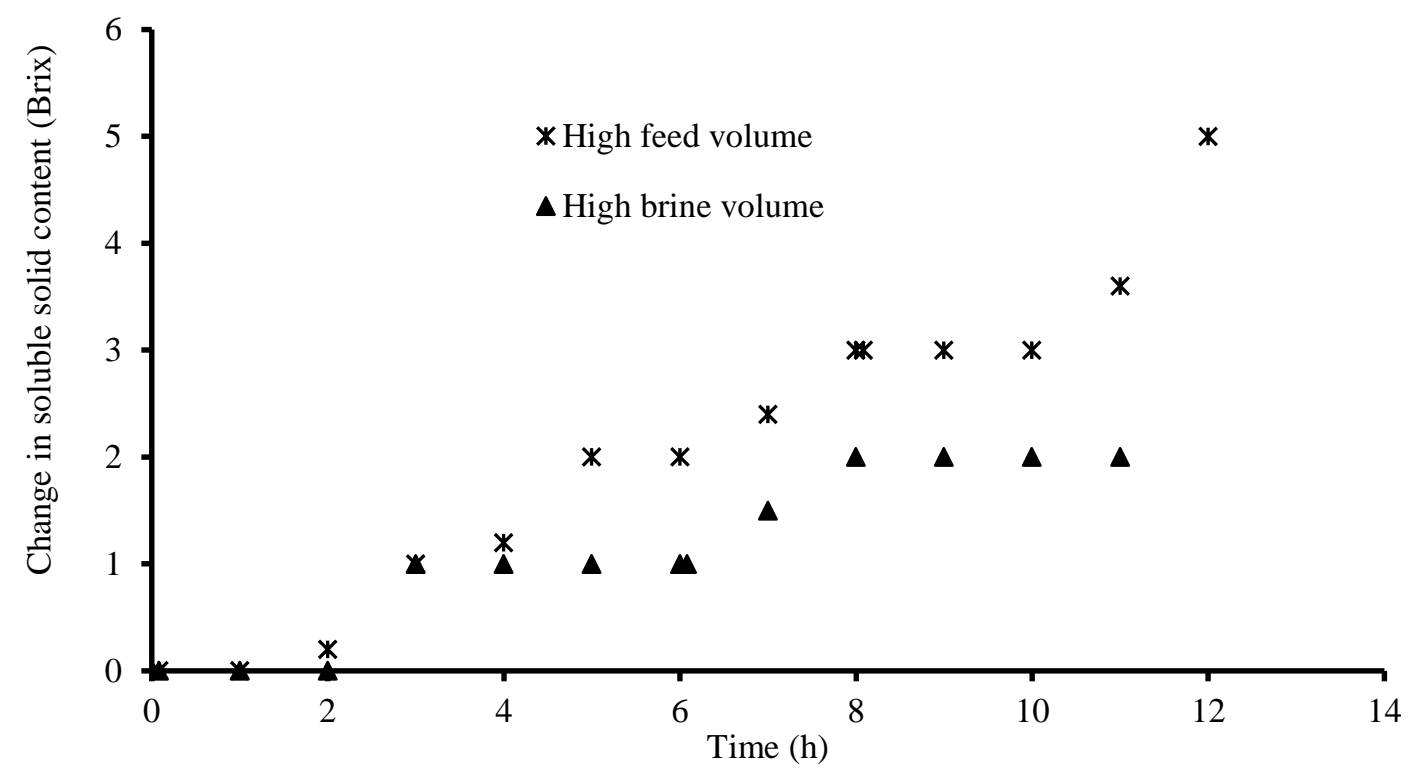

Fig. 5. Effects of feed and brine volumes on changes in soluble solid content of feed during membrane concentration of the red beet juice

Based on the findings, the major decrease in weight of the red beet juice was observed in high brine flow rate, compared to other setups (Fig. 6). In other words, the most increase in SSC of the red beet juice occurred when the brine flow rate was high, compared to other configurations (Fig. 7).

Effects of membrane surface on efficiency of the concentration process: Findings revealed that increased membrane active area increased membrane concentration performance of the red beet juice (Fig. 8). Interestingly, use of membranes with a higher active area $\left(131.75 \mathrm{~cm}^{2}\right)$ remarkably increased TSS content of the juice, compared to that use of membranes with a lower active area (87.83 and 43.91 $\mathrm{cm}^{2}$ ) did, when the process continued for more than three hours. Furthermore, flux of TSS content rise $\left(\mathrm{J}_{\mathrm{f}}\right.$, ${ }^{\circ}$ Brix $\mathrm{m}^{-1} \mathrm{~s}^{-1}$ ) can be considered as an appropriate parameter to assess efficiency of the OD, which can be calculated using Equation 2.

$$
J_{f}=\frac{B I}{A \times t}
$$

Where, BI, A and $\mathrm{t}$ were SSC ( ${ }^{\circ}$ Brix) increase, membrane active area $\left(\mathrm{m}^{2}\right)$ and time (s), respectively. Fig. 9 shows that the flux of TSS content rise was approximately similar to those from experiments with various membrane active areas.

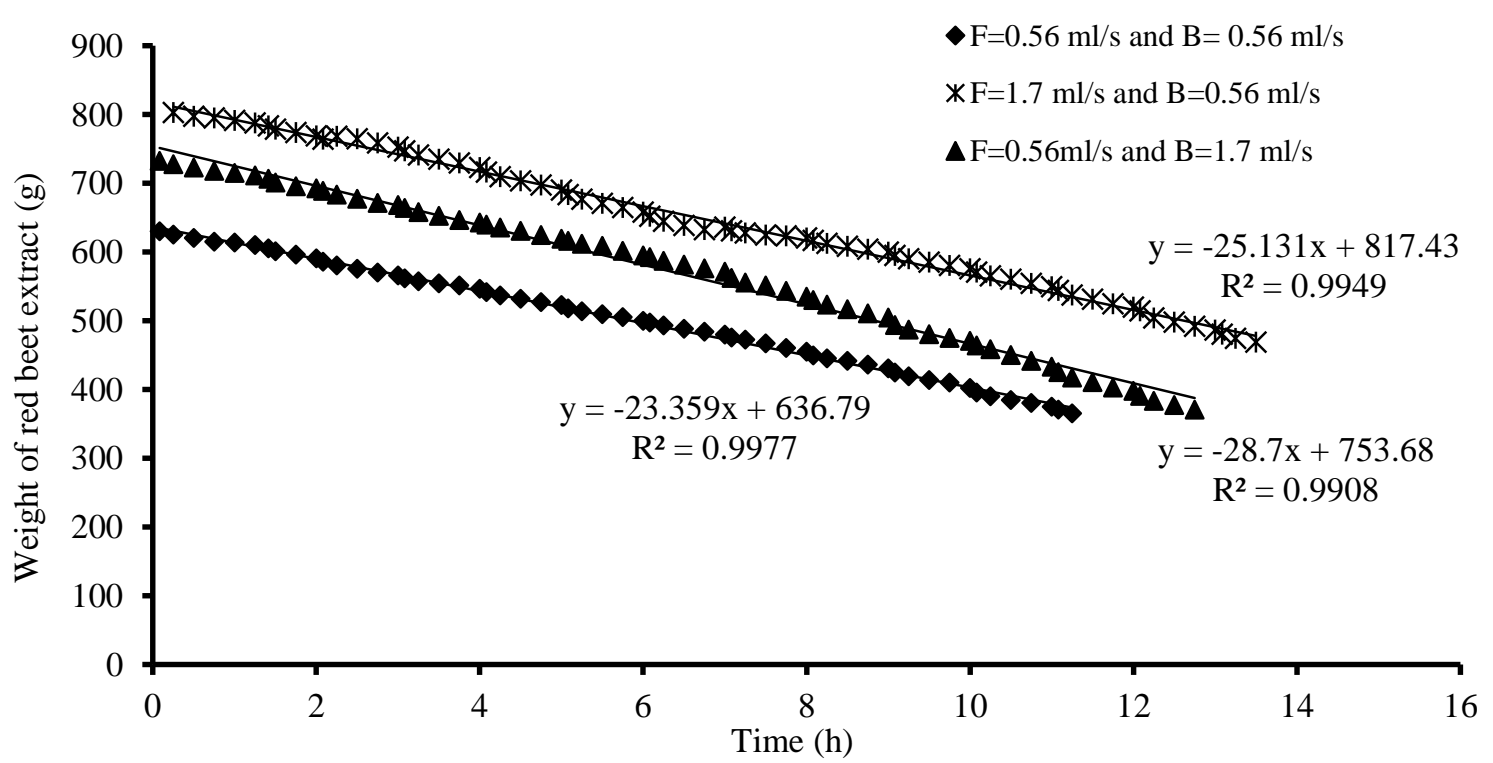

Fig. 6. Effects of the fluid velocity on the weight of feed during membrane concentration of the red beet extract (F, feed flow rate; and $\mathrm{B}$, brine flow rate) 


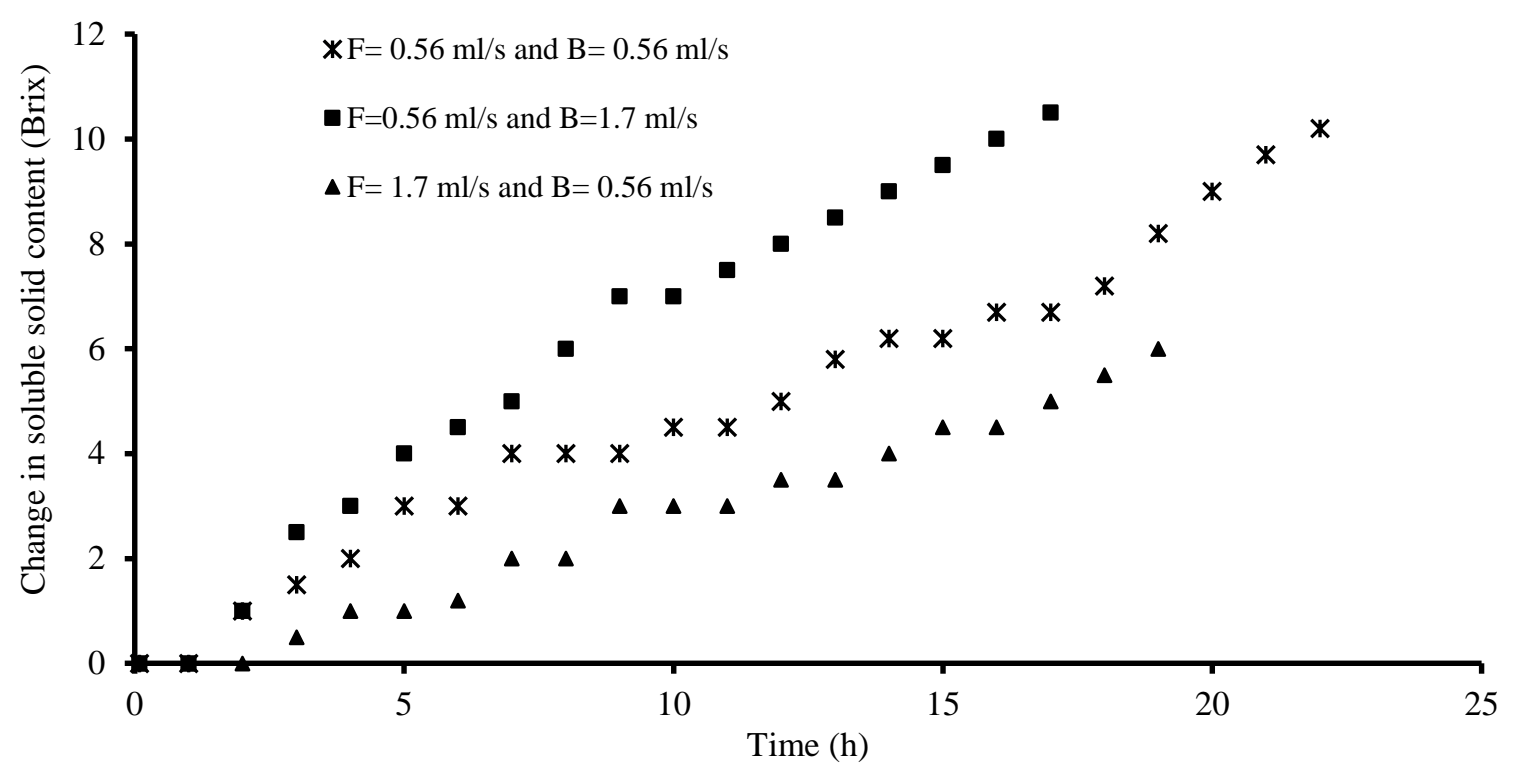

Fig. 7. Effects of the fluid velocity on the soluble solid content of feed during membrane concentration of the red beet extract ( $\mathrm{F}$, feed flow rate; and $\mathrm{B}$, brine flow rate)

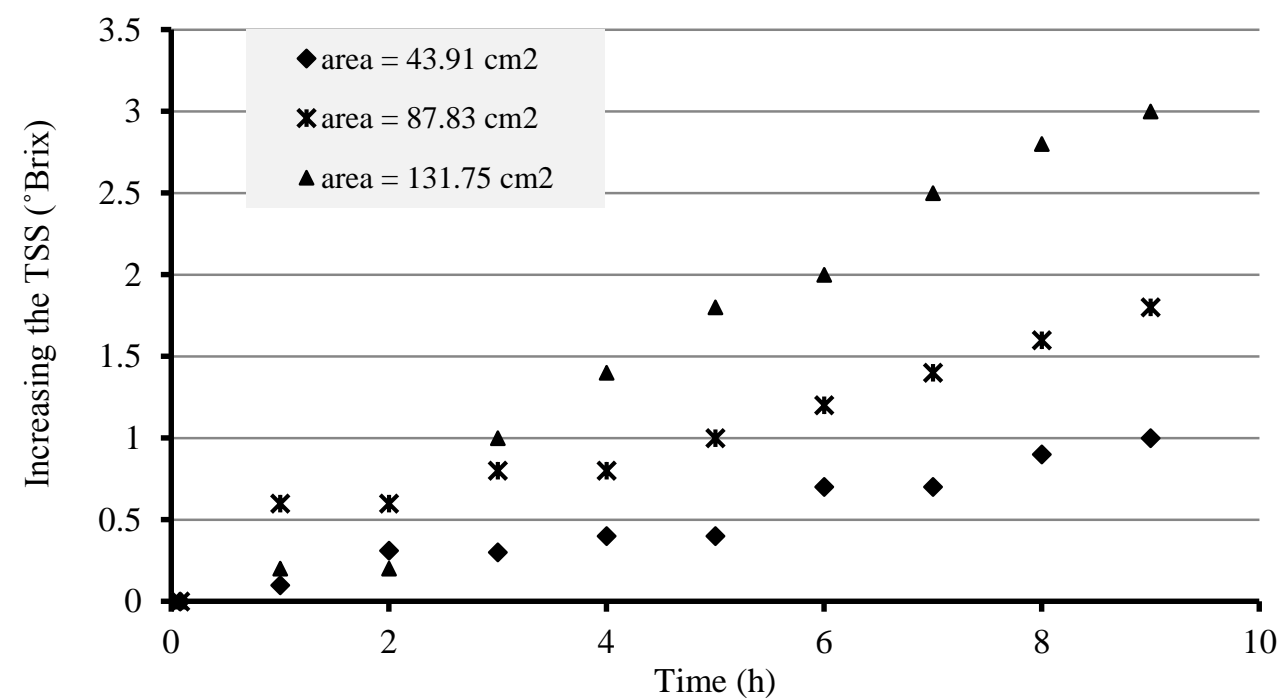

Fig. 8. Effects of the membrane active area on the concentration efficiency of the red beet juice

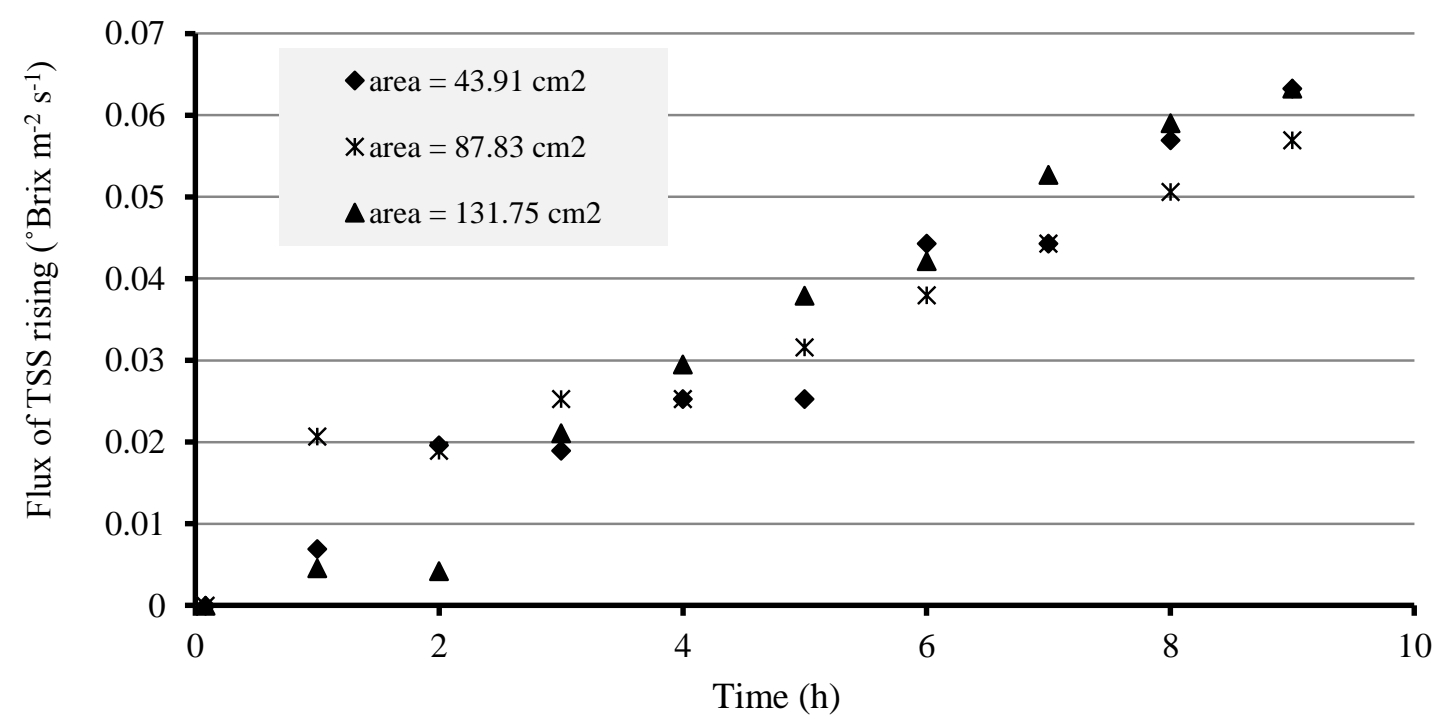

Fig. 9. Flux of the total soluble solid (TSS) rising during membrane concentration of the red beet juice 


\section{Discussion}

In general, a further feed weight reduction was seen in higher volumes of feed and brine, compared to that seen in lower feed and brine volumes. The reduction of feed weight was due to separation of water from red beet juice and its transfer to brine solution via membrane pores. Consequently, membrane concentration efficiency of the red beet juice increased by increasing feed and brine volumes. The high feed volume was drastically effective on increase of the juice SSC at a constant time, compared to the high brine volume. The high brine flow rate included further effects on weight reduction of the red beet juice, compared to other setups. The highest SSC of the red beet juice was observed at the high brine flow rate. Feed and brine particles convectively moved toward the membrane surface and accumulated until an equilibrium between convective and diffusive fluxes was reached. The solute concentration changed from maximum on the membrane surface to minimum in the bulk. However, back transport mechanisms of the small molecules, macromolecules and colloids differ. Large particles and small molecules are back transported by shear-induced diffusion and Brownian diffusion, respectively. Convective flow toward the membrane surface is greater than back-diffusive flow into the bulk solution before the steady state, which is called the concentration polarization (CP) phenomenon. The CP increases density of the rest of component on the membrane surface and accelerates the membrane fouling. Therefore, it acts negatively in membrane processes. Ravindra Babu et al. (17) concluded that $\mathrm{CP}$ in brine side was more dominant than in feed side. As stated previously, the best performance during concentration of the red beet juice occurred when CP in the brine side decreased. Indeed, the current results suggested that increased flow rate in the brine side increased membrane concentration efficiency of the red beet juice via decreasing $\mathrm{CP}$ in the brine side. The membrane active area includes a great effect on membrane concentration performance of the red beet juice. Correspondingly, use of membranes with a higher active area results in a notable increase in TSS content of the juice. Therefore, results from studies, in which various membrane surfaces used for the concentration process, are not comparable. Similarly, roles of membrane morphology and operating conditions on effectiveness of the process have been reported (18).

\section{Conclusion}

In general, concentration process efficiency of the red beet juice can be increased using osmotic distillation by modification of module configuration and process setup. Based on the results from this study, all setups with capability of decreasing concentration polarization improved performance of the concentration process. Furthermore, increased feed volume and decreased brine solution volume increased efficiency of the membrane concentration process. It was found that flow rate enhancement of the brine could increase membrane concentration efficiency of the red beet juice. In conclusion, flux of the total soluble solid content rise can be used as a parameter to show efficiency of the membrane concentration process.

\section{Financial disclosure}

The authors declared no financial interest.

\section{Funding/Support}

This work was financially supported by the University of Tehran.

\section{References}

1. Netzel M, Stintzing FC, Quaas D, Strab G, Carle R, Bitsch R, Bitsch I, Frank T. Renal excretion of antioxidative constituents from red beet in humans. Food Res Int 2005; 38: 1051-1058.

2. Frank T, Stintzing FC, Carle R, Bitsch I, Quaas D, Stra BG, Bitsch R, Netzel M. Urinary pharmacokinetics of betalains following consumption of red beet juice in healthy humans. Pharmacol Res 2005; 52: 290-297.

3. Ravichandran K, Saw NMMT, Mohdaly AAA, Gabr AMM, Kastell A, Riedel H, Cai Z, Knorr D, Smetanska, I. Impact of processing of red beet on betalain content and antioxidant activity. Food Res Int 2013; 50: 670 675 .

4. Ravichandran K, Ahmed AR, Knorr D, Smetanska, I. The effect of different processing methods on phenolic acid content and antioxidant activity of red beet. Food Res Int 2012; 48: 16-20.

5. Stintzing FC, Carle R. Functional properties of anthocyanins and betalains in plants, food, and in human nutrition. Trends Food Sci Technol 2004; 15: 19-38.

6. Cassano A, Jiao B, Drioli E. Production of concentrated kiwifruit juice by integrated membrane process. Food Res Int 2004; 37: 139-148. 
7. Cassano A, Figoli A, Tagarelli A, Sindona G, Drioli E. Integrated membrane process for the production of highly nutritional kiwifruit juice. Desalination 2006; 189: 21-30.

8. Hongvaleerat C, Cabral LMC, Dornier M, Reynes M, Ningsanond $S$. Concentration of pineapple juice by osmotic evaporation. J Food Eng 2008; 88: 548-552.

9. Valdés H, Romero J, Saavedra A, Plaza A, Bubnovich V. Concentration of noni juice by means of osmotic distillation. J Membr Sci 2009; 330: 205-213.

10. Thanedgunbaworn R, Jiraratananon R, Nguyen, MH. Mass and heat transfer analysis in fructose concentration by osmotic distillation process using hollow fibre module. J Food Eng 2007; 78: 126-135.

11. Galaverna G, Silvestro GD, Cassano A, Sforza S. A new integrated membrane process for the production of concentrated blood orange juice: Effect on bioactive compounds and antioxidant activity. Food Chem 2008; 106: $1021-1030$.

12. Cassano A, Conidi C, Timpone R, Avella MD, Drioli E. A membrane-based process for the clarification and the concentration of the cactus pear juice. J Food Eng, 2007; 80: 914-921.
13. Wang L, Min J. Modeling and analyses of membrane osmotic distillation using non-equilibrium thermodynamics. J Membr Sci 2011; 378: 462-470.

14. Cassano A, Drioli E, Galaverna G, Marchelli R, Di Silvestro G., Cagnasso P. Clarification and concentration of citrus and carrot juices by integrated membrane processes. J Food Eng 2003; 57: 153-163.

15. Cassano A, Conidi C, Drioli E. Clarification and concentration of pomegranate juice (Punicagranatum L.) using membrane processes. J Food Eng 2011; 107: $366-373$.

16. Amirasgari N, Mirsaeedghazi H. Non-thermal production of natural betalain colorant concentrate from red beet extract by using the osmotic distillation. Nutrition Food Sci Res 2016; 3(2): 27-34.

17. Ravindra Babu B, Rastogi N, Raghavarao K. Concentration and temperature polarization effects during osmotic membrane distillation. J Membr Sci 2008, 322(1): 146-153.

18. Terki L, Kujawski W, Kujawa J, Kurzawa M, FilipiakSzok A, Chrzanowska E, Madani K. Implementation of osmotic membrane distillation with various hydrophobic porous membranes for concentration of sugars solutions and preservation of the quality of cactus pear juice. $\mathrm{J}$ Food Eng 2018; 230: 28-38. 